\title{
Evolution and resolution of oedema following severe temporary cerebral ischaemia in the gerbil
}

\author{
STUART AVERY, H ALAN CROCKARD, RALPH ROSS RUSSELL \\ From the Cerebral Oedema Research Group, Institute of Neurology, Queen Square, London, UK
}

SUMMARY Regional cerebral blood flow (rCBF) and oedema following profound temporary ischaemia were studied in the gerbil. Ninety-four per cent of animals died within 24 hours of reperfusion; $50 \%$ by 4 hours. Regional differences in oedema (specific gravity method), Evans blue (EB) staining and rCBF (hydrogen clearance technique) occurred. Oedema developed during arterial occlusion, being inversely proportional to residual flow and was markedly exacerbated during reperfusion. Reperfusion hyperaemia was maximal in the parietal and hippocampal regions (ischaemic rCBF $4 \mathrm{ml} 100 \mathrm{~g}^{-1} \mathrm{~min}^{-1}$ ). Oedema was disappearing in all areas by 3 hours of reperfusion and autoregulation returned in the occipital region (mean ischaemia rCBF $8 \mathrm{ml} 100$ $\mathrm{g}^{-1} \min ^{-1}$ ). EB staining and haemorrhage appeared in the thalamus (rCBF $10 \mathrm{ml} 100 \mathrm{~g}^{-1} \mathrm{~min}^{-1}$ ) as oedema was decreasing. It is suggested that the amount of oedema and hyperaemia during reperfusion are dependent on the severity of the ischaemia. Areas of moderate ischaemia (8-10 $\mathrm{ml} 100 \mathrm{~g}^{-1} \mathrm{~min}^{-1}$ ) show little hyperaemia and greater oedema resolution during reperfusion as compared to areas of severe ischaemia (circa $4 \mathrm{ml} 100 \mathrm{~g}^{-1} \mathrm{~min}^{-1}$ ) where there is marked hyperaemia with less oedema resolution. Early in the reperfusion period, oedema is not associated with EB staining and indicates a cytotoxic mechanism. The vasogenic component, with macroscopic haemorrhages and leakage of EB occurs later. In this model it is concluded that the early cytotoxic oedema formation and hyperaemia are phenomena with little bearing on mortality, which correlates better with later vasogenic changes.

Oedema will develop in ischaemic brain tissue and clear thresholds have been demonstrated at which the process develops. ${ }^{2}$ The level of ischaemia at which oedema begins is similar to that at which electrical activity ceases. ${ }^{34}$ In the clinical situation, following a stroke, there may be a subsequent deterioration and the CT scan may demonstrate a brain shift and oedema surrounding the infarct. Following neonatal asphyxia the child's condition may deteriorate after 18-36 hours and recent nuclear magnetic resonance studies suggest that water is accumulating within the brains. There is ample evidence in the literature to demonstrate the time course of oedema which develops following experimental ischaemia ${ }^{5}$ and this is in the same time period noted clinically. Clearly, the process is dynamic and

Address for reprint requests: Mr HA Crockard, FRCS, The National Hospitals for Nervous Diseases, Queen Square, London WC1N 3BG, UK

Received 30 June 1983 and in revised form 12 December 1983. Accepted 22 December 1983 not a static relationship implied in some work: little is known about resolution of oedema.

With reperfusion into the ischaemic area, another variable is introduced. The blood brain barrier may have been damaged. Intravascular fluid and "oedemagenic substances" may leak into the damaged cerebral tissue causing further deterioration. It is a situation with clear clinical implications such as that which would occur following a cardiac arrest, or cerebral embolus or surgical procedure. Iannotti and Hoff, ${ }^{6}$ studying reperfusion oedema in the gerbil, have shown that in areas of moderate ischaemia ( $\mathrm{rCBF}$, greater than $10 \mathrm{ml} 100 \mathrm{~g}^{-1} \mathrm{~min}^{-1}$ ) oedema will rapidly resolve following restoration of flow. In severely ischaemic regions, less than $7 \mathrm{ml}_{100 \mathrm{~g}^{-1}}$ $\mathrm{min}^{-1}$, oedema is aggravated during reperfusion. Whether the development and resolution of oedema has anything to do with mortality and morbidity has yet to be decided.

We have used a model of bilateral carotid occlusion in gerbils which reliably produces profound ischaemia in the anterior two-thirds of the cerebral 
hemispheres, in order to study variations of flow and oedema, and correlated them with clinical survival following carotid occlusion. We have chosen a model of certain mortality to assess the effects of reperfusion through non viable cerebral tissue and to correlate this with our studies in brain tissue prostaglandins. ${ }^{7}$ In this paper we describe the clinical effects and cerebral vascular changes consequent on 1 hour of bilateral carotid occlusion.

\section{Methods and materials}

Cerebral ischaemia was produced by bilateral common carotid occlusion, with or without reperfusion in 132 male mongolian gerbils (weight range 42-70 g). All animals received intraperitoneal pentobarbitone anaesthesia (60 $\left.\mathrm{mg} \mathrm{kg}^{-1}\right)$, with supplementation as necessary. Surgery was performed with an operating microscope, the common carotid arteries being carefully isolated and a sling passed under each vessel to ensure simultaneous bilateral occlusion with Scoville Lewis aneurysm clips. Temperature was maintained at $37^{\circ} \mathrm{C}$ by heating blanket and lamp. In animals undergoing rCBF studies, a femoral artery catheter gave access for continual blood pressure (MABP) monitoring and intermittent blood gases.

The experimental protocol was divided into four groups:-

Group A-Mortality and morbidity studies (34 gerbils).

Group B-Macroscopic morphology (27 gerbils plus 20 from Group A).

Group C-Specific gravity studies (53 gerbils, including some from Group D).

Group D-Regional cerebral blood flow (rCBF) (21 gerbils).

\section{Group A: Mortality and morbidity}

Thirty-four gerbils were observed following 60 minutes of temporary bilateral cerebral ischaemia. Note was made of paresis, seizures, conscious level and time of death. When breathing stopped, fixation-perfusion was carried out by infusion of saline, followed by $10 \%$ formalin, through the left ventricle. Brains were removed and immediately examined macroscopically after 12 coronal slices had been cut.

\section{Group B: Macroscopic and morphological changes} Evans blue was given as a bolus intravenously $(0.05 \mathrm{ml}$ of $2 \%$ solution in saline $\mathrm{pH} \mathrm{7.4)} 30$ minutes after application of the clip. Twenty-seven animals were sacrificed at set times to assess the evolution of Evan's blue (EB) staining. These reperfusion time intervals (with numbers of animals) of 60 minutes (9), 120 minutes (10), and 180 minutes (8) were taken following the standard hour of ischaemia. Animals were kill-perfused with $10 \%$ formalin and the presence of EB discoloration and/or haemorrhages noted.

Group C: Specific gravity (SG)

Regional specific gravity was measured in 53 gerbils according to the techniques of Marmarou et al. ${ }^{89}$ Slight adjustments were made to the column calibration span to allow for gerbil grey matter. Gerbils were sacrificed by decapitation, the brain being rapidly removed and placed in a kerosene filled petri dish. Six pieces $1 \mathrm{~mm}^{3}$ were cut from each of eight regions; frontal, parietal, occipital, corpus striatum, hippocampus, thalamus, cerebellum and pons. The linearly inhomogenous columns of kerosene and bromobenzene were calibrated with six known SG standards. Only columns with a linear correlation coefficient (r) better than 0.9950 were accepted for use. The samples were allowed to settle for 3 minutes, when the exact level was noted. The following groups were examined (number in brackets equals number of animals); control, with anaesthetic, but no surgery (4). Sham sampled 2 hours after operation, but without occlusion (4), 60 minutes ischaemia only (4), 120 minutes ischaemia only (4). The following reperfusion periods were chosen after 1 hour of ischaemia; 5 minutes (4), 30 minutes (8), 60 minutes (8), 120 minutes $(8)$, and 180 minutes $(9)$.

\section{Group D: Regional cerebral blood flow ( $r C B F$ )}

rCBF was measured by the hydrogen $\left(\mathrm{H}_{2}\right)$ clearance technique of Aukland et al (1964), ${ }^{10}$ with modifications ${ }^{112}$ in 21 gerbils. Burr holes were made in the skull and six platinum-iridium teflon coated electrodes $(175 \mu \max$. diameter), with $1 \mathrm{~mm}$ of the tip exposed, were positioned with micro-manipulators and fixed with methylmethacrylate cement. Placement was confirmed after death: there was a mixture of cortical and deep electrode placements in each animal so that all sites were studied in the group as a whole. Flow was calculated by the initial slope technique. The slope of the log height-time curve, multiplied by 100 gave the rCBF in $\mathrm{ml} 100 \mathrm{~g}^{-1} \mathrm{~min}^{-1} \cdot 4$ (See section on Validation of Technique.) Zero flow was defined as a lack of $\mathrm{H}_{2}$ clearance, with no change upon further $\mathrm{H}_{2}$ administration. Hyperaemia was defined as a rCBF measured during reperfusion that was greater than the control value. All rCBF studies had arterial blood gases measured prior to occlusion, and once or twice during reperfusion (total volume less than $410 \mu \mathrm{l}$ ).

Validation of Technique Duplicate flows showed a $\pm 6 \%$ reproducibility. The semilogarithmic plots were monoexponential in the gerbil. ${ }^{12} 2$ In $82 \%$ of flow estimations, the coefficient of determination $\left(r^{2}\right)$ for the exponential regression was $\geqslant 0.9950$, between 0.9900 and 0.9949 in $12 \%$ and $\geqslant 0.9850$ and $<0.9899$ in less than $5 \%$. Fewer than $1 \%$ were non linear and in all these a brief variation in MABP was noted (for example seizure). The poorest values of $\mathbf{r}^{2}$ were seen with slow flows in the immediate reperfusion phase.

\section{Analysis}

Chi squared testing with the Yates correction for continuity was used in the analysis of the mortality data, incidence of haemorrhages and Evans blue staining. Flow and oedema studies were compared by the student's $t$ test (two tailed, unpaired). Results are expressed as means \pm one standard deviation.

\section{Results}

Mean arterial blood pressure (MABP) was $61 \pm 10$ 


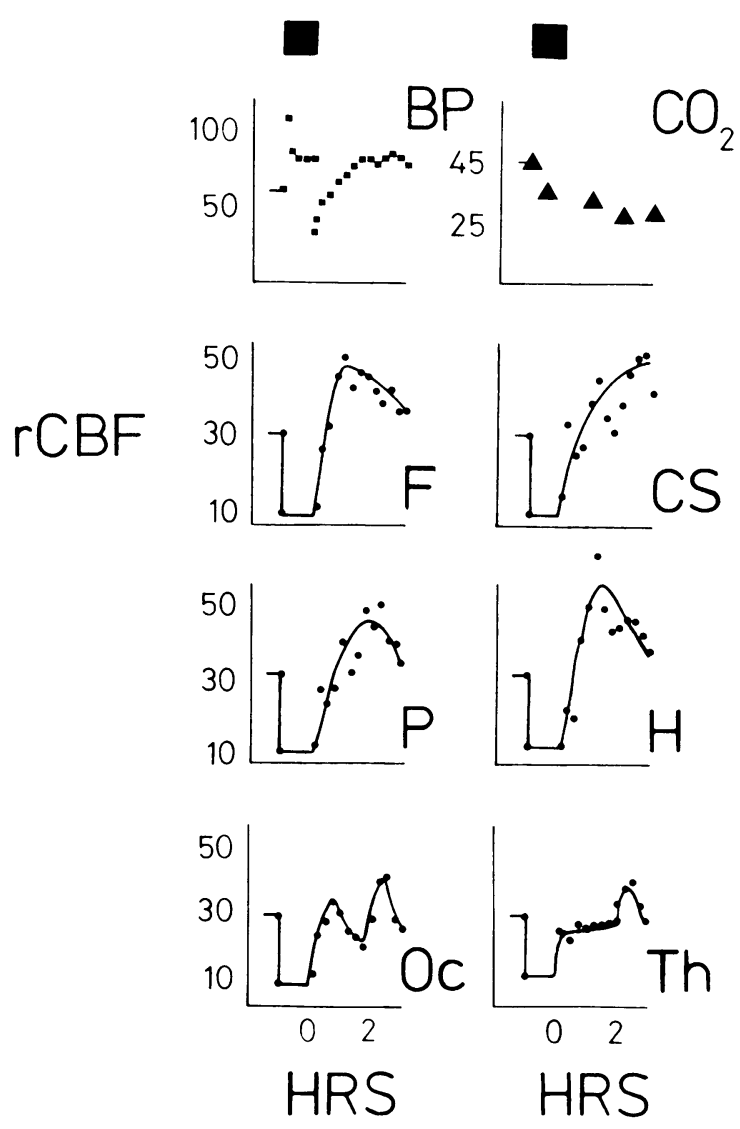

Fig 1 The mean systemic blood pressure $(B P)$ and arterial blood $\mathrm{CO}_{2}\left(\mathrm{CO}_{2}\right)$ during regional cerebral blood flow measurements are shown prior to, during and for three hours following $60 \mathrm{~min}$ bilateral carotid occlusion. The black box represents the occlusion time. The mean values from 21 animals are shown. During occlusion there was an increase in BP and a temporary fall with removal of the clips. The animals hyperventilated in the reperfusion period producing a reduction in $\mathrm{CO}_{2}$. There was significant hyperaemia (I SEM greater than control flows) in all but the thalamus (Th). The time course is similar to the changes in $S G$.

$r C B F=$ regional cerebral blood flow; $F=$ frontal; $P=$ parietal; $O_{C}=$ occipital; $(s=$ corpus striatum; $H=$ hypothalamus; $T h=$ thalamus.

O hours = beginning of reperfusion

$\mathrm{mm} \mathrm{Hg}(\mathrm{n}=21)$, and rose sharply with clipping (fig 1), but fell to a plateau above resting levels for the remainder of carotid occlusion. Blood pressure plunged to a nadir $(40 \mathrm{~mm} \mathrm{Hg})$ within 2 minutes of clip release, recovering by 5 minutes and reaching control levels by 30 minutes. MABP stabilised at 80

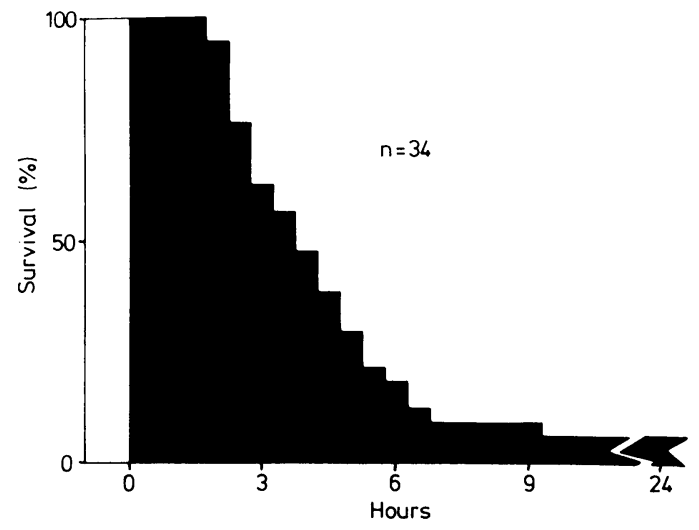

Fig 2 The survival following 1 hour's bilateral carotid occlusion followed by reperfusion is graphically displayed. Half of the animals had succumbed between 3 and 4 hours of reperfusion and $94 \%$ were dead at 24 hours.

$\mathrm{mm} \mathrm{Hg}$ for the last 90 minutes of protocol. Brief transients of MABP were recorded with convulsions. $\mathrm{PaCO}_{2}$ was $43.9 \pm 5.0 \mathrm{~mm} \mathrm{Hg}$ with a fall during the hyperventilation associated with carotid occlusion; statistically the levels did not alter subsequently.

GROUP A: MORTALITY AND MORBIDITY

In the 34 animals there was a $50 \%$ mortality by 4 hours of reperfusion, and only $6 \%$ survived to 24 hours (fig 2). During the second hour of reperfusion. signs of severe neurological damage were invariable, with circling or hemiparetic behaviour, abnormal posture and irritability to sound. Fits, both focal and generalised, began with arousal in the second hour of reperfusion in all animals recovering consciousness.

\section{GROUP B: MACROSCOPIC MORPHOLOGICAL CHANGE}

This group consisted of animals followed to death (Group A) and others killed at predetermined intervals. The earliest EB staining was seen at 60 minutes of reperfusion in one of nine gerbils. The prevalence of EB was $50 \%$ by 120 minutes and more pronounced in degree at 180 minutes. Leakage was usually bilateral and symmetrical, being most marked in the thalamus and lateral geniculate bodies. The parietal, hippocampal, lateral mid-brain and occipital areas were less often affected. Of the animals showing EB staining, half had haemorrhages in the thalamus. Haemorrhages were thalamic except for two cases (central pontine grey and bilateral hippocampal). Haemorrhage and EB occurred in similar proportions $\left(\mathrm{X}_{\mathrm{C}}{ }^{2} \mathrm{~N} . \mathrm{S}\right.$.) (table), 
Table Incidence of Evans blue staining and haemorrhage following reperfusion after 60 carotid occlusion

\begin{tabular}{lrrl}
\hline & \multicolumn{3}{c}{ Reperfusion time } \\
\cline { 2 - 4 } & $1 \mathrm{hr}$ & $2 \mathrm{hrs}$ & $3 \mathrm{hrs}$ \\
\hline Evans Blue (EB) & $11 \%$ & $50 \%$ & $62 \%$ \\
Haemorrhage (H) & $0 \%$ & $22 \%$ & $28 \%$ \\
H + EB & $0 \%$ & $0 \%$ & $25 \%$ \\
H - EB & $0 \%$ & $10 \%$ & $13 \%$ \\
\hline
\end{tabular}

The incidence of Evans blue staining (noted on inspection) and haemorrhage into the deep grey structures in given. There is no gross EB staining before one hour or reperfusion and no haemorrhages until two hours. However, there is no obvious relationship between $\mathrm{EB}$ staining and the presence of haemorrhages.

$\mathrm{H}+\mathrm{EB}$ Haemorrhage and Evans Blue in same animal.

H - EB Haemorrhage without Evans Blue in same animal.
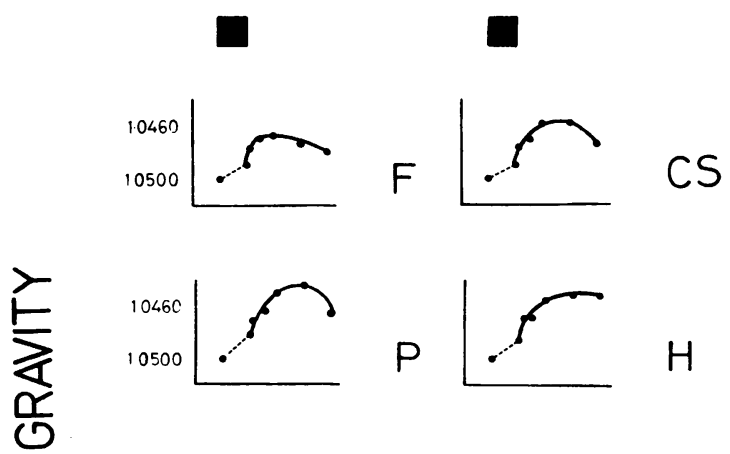

$\mathrm{H}$
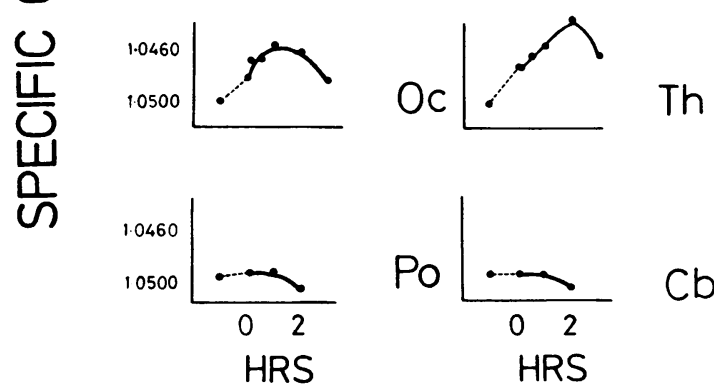

Fig 3 Regional specific gravity measurements before, and after 60 min bilateral carotid occlusion are shown. The results are mean data obtained from groups of at each time point. The numbers are given in the text but was between 4 and 9 for each time point. $X$-axis displays sham animals, their dotted line shows changes during occlusion (Black Box) and solid line indicates regional specific gravity patterns during three hours of reperfusion. In the carotid territory there was significant increase in water, maximal at $1-2 h$, which then subsided. In the non-ischaemic vertebrobasilar territory (Po and $\mathrm{Cb}$ ) there is no change in specific gravity.

$S G 1.0500=$ normal. $1.0460=$ oedema .

$F=$ frontal $; P=$ parietal; $O c=$ occipital; $C s=$ corpus striatum; $H=$ hypothalamus; $T h=$ thalamus; $P o=$ pons; $\mathrm{Cb}=$ cerebellum. although EB was more commonly bilateral, and tended to manifest earlier. Focal seizures did not correlate with asymmetries of EB or haemorrhage.

\section{GROUP C: SPECIFIC GRAVITY}

During the 60 minutes of ischaemia all areas in the carotid vascular territory showed a significant fall in SG (increase in water). There was further and more rapid accumulation of oedema within 5 minutes of clip removal (fig 3), which was greatest in areas with more severely impaired occlusion flows. By 5 minutes of reperfusion the degree of oedema was very similar throughout the brain and it continued to increase for a further 60 minutes. The thalamus showed the most pronounced oedema during the ischaemic phase and, uniquely, did not show an increase in water accumulation during reperfusion. All areas, apart from the hippocampus, showed a return towards normal SG in the third hour of reperfusion. Resolution of oedema was greatest in the areas of least severe occlusion ischaemia and, for example, in the occipital area where the SG returned to control levels (figs 1 and 3). Despite simultaneous bilateral occlusion and release of the arteries, differences in SG were found between the left and right hemispheres, being most pronounced in the parietal areas. There was no change in the SG in the pons or cerebellum following ischaemia and reperfusion.

GROUP D: REGIONAL CEREBRAL BLOOD FLOW In spontaneously breathing intact animals under barbiturate anaesthesia, the mean CBF was $29.6 \mathrm{ml}$ $100 \mathrm{~g}^{-1} \mathrm{~min}^{-1}$ (215 electrodes). Mean rCBF ranged from 23 (corpus striatum) to 38 (parietal) $\mathrm{ml} 100$ $\mathrm{g}^{-1} \min ^{-1}$. Clipping produced a marked fall in rCBF to $3 \mathrm{ml} 100 \mathrm{~g}^{-1} \mathrm{~min}^{-1}$ in the forebrain and $10 \mathrm{ml} 100$ $\mathrm{g}^{-1} \min ^{-1}$ in the thalamus and occiput. Following clip release, there was no significant mean flow change at 5 minutes. Regions of zero flow were seen occasionally in the forebrain during the early reperfusion phase, but these did not persist. Hyperaemia was then seen in all areas, with regional variations; flow returned to control values by 3 hours of reperfusion. Hyperaemia immediately after clip release was seen in the thalamus of only one animal. Hyperaemia was most marked in areas where the ischaemic flow was rCBF of $4.1 \pm 1.5 \mathrm{ml} 100 \mathrm{~g}^{-1}$ $\min ^{-1}$, (parietal area, fig 1). There was no direct relationship between peak hyperaemia and peak oedema. MABP and rCBF in the first and third hour of reperfusion showed a passive pressure-flow relationship, suggesting a loss of autoregulation (fig 4). Uniquely, in the occipital area there was a change in this relationship by the third hour of reperfusion. The difference between the slopes of the two regres- 


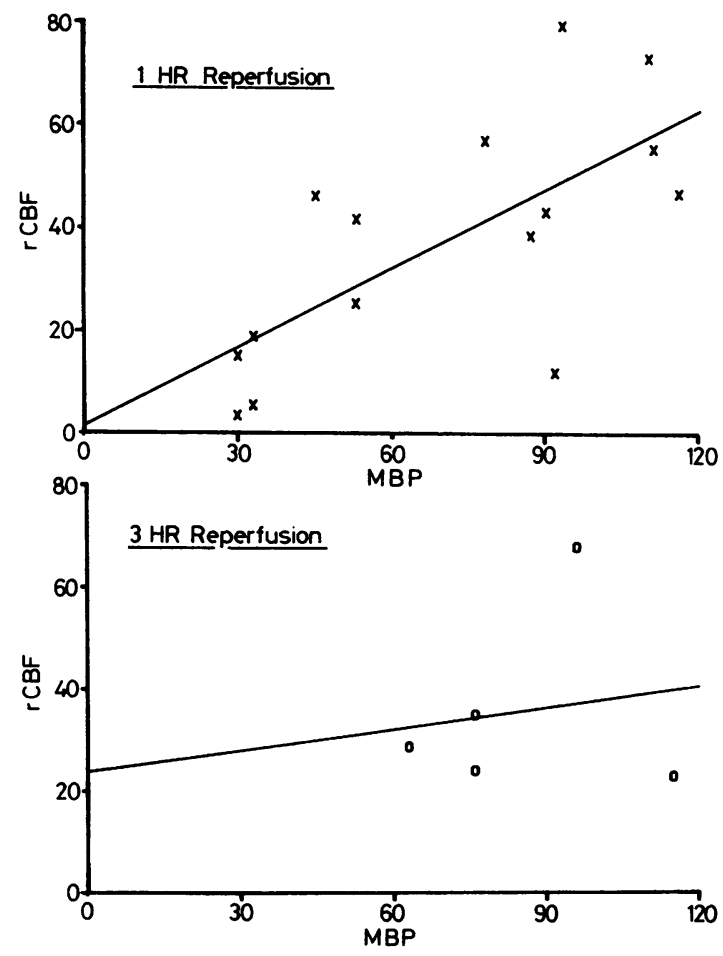

Fig 4 Autoregulation in response to induced blood pressure changes is shown during the reperfusion phase in the occipital regions. Blood pressure changes and corresponding regional cerebral blood fiow changes 1 hour and 3 hours after the removal of the occlusive carotid clips are shown. A linear regression analysis of the flow and blood pressure results allowed construction of the graphs. There is a passive pressure flow relationship in the first hour of occlusion, but by the third hour reperfusion correlation had altered. The mean $\mathrm{CCBF}$ in the occipital region was 10 $\mathrm{ml} \mathrm{gm}^{-1} \mathrm{~min}^{-1}$ during occlusion. In the frontal and parietal areas where occlusive flows were lower, autoregulation did not return.

sion lines suggested that autoregulation had been re-established in this area. $y=0.51 x^{+} 1.36 ; r^{2}=0.5$ $(N=15)$ at one hour of reperfusion and $y=0.28 x$ ${ }^{+} 16 \cdot 1 ; \mathrm{r}^{2}=0.2(\mathrm{~N}=5)$ at three hours of reperfusion.

\section{Discussion}

The gerbil has been used as a model of cerebral ischaemia because the hemispheric blood supply is from the carotid arteries with incomplete anastomosies of the circle of Willis. Microvascular anastomosies exist but these cannot develop sufficiently to sustain life. ${ }^{13}$ Bilateral ligation results in $100 \%$ mortality within 4 hours, whereas a unilateral liga- tion results in a mortality of $20-65 \% . .^{1415}$ The reason for the wide range in mortality with a unilateral ligation is the side to side variations in the circle of Willis which have been elegantly demonstrated by Tamura et al. ${ }^{16}$ With temporary bilateral occlusion there is a mortality which varies with the period of occlusion. Fifteen minutes of bilateral occlusion rarely causes death; 30 minutes occlusion produces a $40 \%$ mortality at 24 hours. ${ }^{15}$ Restoration of circulation after 1 hour's carotid occlusion results in $94 \%$ mortality within 24 hours. These results are similar from different laboratories and under different anaesthetics, ${ }^{17}$ and provide a crude but reproducible index of the depth and duration of ischaemic tolerance. They provide a baseline for the assessment of possible therapeutic agents aimed at minimising the effect of the ischaemic insult.

Histology of a unilateral occlusion has been well described ${ }^{18}$ and has shown maximal damage within the thalamus and surrounding deep grey structures. In our bilateral occlusion animals, with reperfusion, there was maximal damage to blood vessels within the thalami with frank haemorrhage and Evans blue leakage. This is in spite of a blood flow during ischaemia comparable to the occipital region which appears to survive and in which autoregulation returns. Evans blue leakage appears to precede signs of frank haemorrhage in the area and both haemorrhage and Evans blue leakage are related to the length of the occlusive period, for we have not noted haemorrhages with occlusions lasting $\mathbf{3 0}$ minutes or less and Evans blue leakage is also less common with shorter occlusion times. These changes therefore would also appear to be related to duration of ischaemia.

To standardise the model, we have used adult male gerbils. Mortality is independent of sex in bilateral ischaemia, ${ }^{17}$ but uniformity in weight and maturity is important to exclude animals more resistant (young), or more sensitive (old) to ischaemia. We have chosen intraperitoneal barbiturate anaesthesia as it provides a reliable stable preparation even though there may be some reduction in mean $\mathrm{CBF}$ and an elevation in $\mathrm{pCO}_{2}$. Its possible cerebral protective property ${ }^{1920}$ is another source of artefact but as the technique is unchanged in all our studies the error introduced by this form of anaesthesia theoretically should be constant.

With occlusion, we have previously shown that there is an increase in cortical water as judged by a decrease in specific gravity following carotid occlusion. $^{2}$ In this study we have extended our observations to the deep grey matter, cerebellum and pons. In the thalamus there is a progressive accumulation during ischaemia, but no change in specific gravities in the pons or cerebellum. During the restoration of 
flow there is a rapid accumulation of water which reaches a peak at 1-2 hours following the occlusion and then subsides. There is no change in water content during reperfusion in the pons and cerebellum where there was no ischaemia and none in the thalamus where there was no hyperaemia. Maximal resolution of oedema occurs in the occiput which has the highest cortical flow and in which autoregulation returned. The changes in specific gravity in general mirror the regional blood flow changes and the question arises as to whether the changes in specific gravity are real or artefacts due to an increase in local cerebral blood volume during the hyperaemia. This cannot be excluded in our experiments, nevertheless, our results are in broad agreement with those who have used differing methods for assessing water accumulation such as wet weight/dry weight ${ }^{18}$ and isotopes. ${ }^{21}$ The specific gravity technique assumes that there is no change in tissue solids during the measurements. If there is protein extravasation, a correction factor has been applied in the cat. ${ }^{9}$ We have been unable to apply the correction factor in gerbils, perhaps because of the distribution of grey and white matter within the gerbil brain. We do not consider that a change in tissue solids would significantly contribute to an error in our result prior to the leakage of Evans blue and a protein marker. The question arises as to whether the specific gravity changes are due to regional changes in blood volume. Shigeno et al $^{22}$ have quantified this artefact and the magnitude of our changes are well in excess of those produced by blood volume. Our own studies on gerbils (unpublished) confirm this. Thus, the changes in specific gravity in the first hour of reperfusion would be generally reliable and in the areas where there was no Evans blue staining might be reliable for the total period of observation. The accumulation and resolution of oedema illustrates the dynamic situation that pertains following restoration flow. Preliminary studies $^{6}$ showed that if the ischaemic flow was above $10 \mathrm{ml} 100 \mathrm{~g}^{-1} \mathrm{~min}^{-1}$, then the oedema accumulating during ischaemia would be resolved within an hour of reperfusion. In areas where the flow was less than $7 \mathrm{ml} 100 \mathrm{~g}^{-1} \mathrm{~min}^{-1}$ there was an increase in oedema after 1 hour's restoration of flow. These time course studies demonstrate that within the severely ischaemia tissue there is resolution of the oedema after the initial increase associated with reperfusion. In the occipital regions with ischaemic flows in the region of $8 \mathrm{ml} 100 \mathrm{~g}^{-1} \mathrm{~min}^{-1}$ a borderline situation exists, and while the oedema resolves it does so more slowly in less ischaemic tissue.

The Evans blue extravasation demonstrating a loss of barrier to protein bound dye was later (1 hour after reperfusion), confined to the deep grey structures and over the short period of our reperfusion studies, progressive. It appeared in the same time course as the animal mortality and the question arises as to whether these observations are causally related or mere coincidence. Further work will be required to elucidate this.

In this study we have extended our observations on the regional cerebral blood flow, judged by hydrogen clearance technique in the gerbil. Flows in the

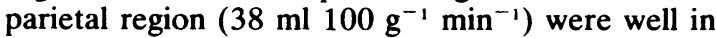
accord with previous results. During bilateral occlusion, flow fell to practically zero in the frontal region with a gradation towards the occipital area. The thalamus had a surprisingly high flow but this is because of the contribution of the posterior circulation to that area. With restoration and flow there was a generalisation of hyperaemia which was maximal in areas with flow in the region of $4 \mathrm{ml} 100$ $\mathrm{g}^{-1} \min ^{-1}$. In the frontal region, there was less hyperaemia than we anticipated and this may have been due to areas of microscopic impaired reperfusion. The hyperaemia corresponded roughly to the intensity and duration of the ischaemia, as reported by others. ${ }^{22}{ }^{23}$ It was interesting that there was the maximal oedema in areas where the ischaemic flow was $4 \mathrm{ml} 100 \mathrm{~g}^{-1} \mathrm{~min}^{-1}$, in the same sort of values at which we noted maximal oedema during ischaemia ${ }^{2}$ and from which areas of intravascular potassium extravation has been reported. ${ }^{24}$

Autoregulation is lost in all regions where flow is less than $13-15 \mathrm{ml}^{100^{-1}} \mathrm{~min}^{-1}$ in the primate, ${ }^{25}$ and in the gerbil Iannotti and $\mathrm{Hoff}^{6}$ have demonstrated a similar finding. In these studies we have shown that in the occipital region with.a mean flow of $8 \mathrm{ml} 100$ $\mathrm{g}^{-1} \min ^{-1}$, autoregulation returns 3 hours after the restoration of flow and this would suggest that in areas where the flow during ischaemia is above $8 \mathrm{ml}$ $100 \mathrm{~g}^{-1} \mathrm{~min}^{-1}$ autoregulation ultimately will return. We have also shown that in the same region, oedema will resolve and it might be concluded that even with this prolonged period of cerebral ischaemia ischaemic flow above $8 \mathrm{ml} 100 \mathrm{~g}^{-1} \mathrm{~min}^{-1}$ might be tolerated with ultimate recovery.

What conclusions might be drawn from our results? Firstly, that in ischaemic tissue there is a reproducible relationship between ischaemic blood flow and the development of oedema. During reperfusion, however, the situation is a dynamic one and while there is a rough correlation between the hyperaemia and the extent of cytotoxic oedema, the relationship is less close. The accumulation and resolution of oedema extends to tissues which will not survive and the time scale of the accumulation and resolution of cytotoxic oedema does not correlate with the time of the animal's death. The latter is more closely related to signs of blood brain barrier 
and vascular endothelial damage. Lastly, we have shown that autoregulation will return in tissues where the flow is above $8 \mathrm{ml} 100 \mathrm{~g}^{-1} \mathrm{~min}^{-1}$ for 1 hour and, with the exception of the thalamic area for which there may be local exceptional circumstances, this level of ischaemic flow might be compatible with ultimate survival and return to normal function.

We thank Professor RW Gilliatt for use of facilities, and Miss Kathryn Allen for technical assistance.

This work was supported by The National Fund for Research into Crippling Diseases and The Brain Research Trust.

\section{References}

' Symon L, Branston NM, Chickovani O. Ischaemic brain edema following middle cerebral artery occlusion in baboons: relationship between regional cerebral water content and blood flow at 1 to 2 hours. Stroke 1974;10:184-91.

${ }^{2}$ Crockard A, Iannotti F, Hunstock AT, Smith RD, Harris RJ, Symon L. Cerebral blood flow and edema following carotid occlusion in the gerbil. Stroke 1980;11:494-8.

${ }^{3}$ Branston NM, Symon L, Crockard HA. Recovery of cortical evoked response following temporary MCA occlusion in baboons: relation to local blood flow and $\mathrm{pO}_{2}$. Stroke 1976;7:151-7.

${ }^{4}$ Young W. $\mathrm{H}_{2}$ clearance measurement of blood flow: a review of technique and polarographic principles. Stroke 1980;11:552-64.

${ }^{5}$ Klatzo I. Neuropathological aspects of brain edema. $J$ Neuropath Exp Neurol 1967;26:1-14.

${ }^{6}$ Iannotti F, Hoff JT. Ischemic brain edema with and without reperfusion: an experimental study in gerbils. Stroke 1983;14:4-7.

${ }^{7}$ Bhakoo KK, Crockard HA, Lascelles PT, Avery SF. Prostaglandin synthesis and oedema formation during reperfusion following experimental brain ischaemia in the gerbil. Stroke (in press).

${ }^{8}$ Nelson SR, Mantz M-L, Maxwell JA. Use of specific gravity in the measurement of cerebral edema. J Appl Physiol 1971;30:268-71.

${ }^{9}$ Marmarou A, Poll W, Shulman K, Bhagavan H. A simple gravimetric technique for measurement of cerebral edema. J Neurosurg 1978;49:530-7.

${ }^{10}$ Aukland K, Bower BF, Berliner RW. Measurement of local blood flow with hydrogen gas. Circ Res 1964;14:164-87.

" Pasztor E, Symon L, Dorsch NWC, Branston NM. The hydrogen clearance method in assessment of blood flow in cortex, white matter and deep nuclei of baboons. Stroke 1973;4:556-67.

12 Heiss W-D, Hayakawa T, Waltz AG. Patterns of changes of blood flow and relationships to infarction in experimental cerebral ischaemia. Stroke 1976;7:454-9.

${ }^{13}$ Payan HM, Conrad JR. Carotid ligation in gerbils. Stroke 1977;8:194-6.

${ }^{14}$ Levine S, Payan H. Effects of ischaemia and other procedures on the brain and retina of the gerbil (Meriones unguiculatus). Exp Neurol 1966;16:25562.

${ }^{15}$ Harrison MJG, Brownbill D, Lewis PD, Ross Russell RW. Cerebral edema following carotid artery ligation in the gerbil. Arch Neurol 1973;28:389-91.

${ }^{16}$ Tamura A, Horizoe H, Fukuda T. Relationship of cerebral vasculature to infarcted areas following unilateral common carotid artery ligation in the mongolian gerbils. J CBF Metab 1, Suppl 1, 1981; S194-S195.

17 Jarrott DM, Domer FR. A gerbil model of cerebral ischaemia suitable for drug evaluation. Stroke 1980;11:203-9.

${ }^{18}$ Ito U, Spatz M, Walker JT Jnr, Klatzo I. Experimental cerebral ischaemia in mongolian gerbils. I. Light microscopic observations. Acta Neuropathol 1975;32: 209-23.

${ }^{19}$ Smith AL, Hoff JT, Nielson SL, Larson CP. Barbiturate protection in acute focal cerebral ischaemia. Stroke 1974;5:1-7.

${ }^{20}$ Michenfelder JD, Milde JH, Sundt TM Jnr. Cerebral protection by barbiturate anaesthesia use after middle cerebral artery occlusion in Java monkeys. Arch Neurol 1976;33:345-50.

${ }^{21}$ Go KG, Houthoff HJ, Huitema S, Spatz M. Protein tracer permeability of the blood-brain barrier after cerebral ischemia in gerbils. Comparison of endogenous and exogenous tracers. In: Proceedings of the 5th International Symposium on Brain Edema. New York Plenum Press 1984.

${ }^{22}$ Shigeno T, Brock M, Shigeno S, Fritschka E, CervosNavarro J. The determination of brain water content. Microgravimetry versus dry weight method. $J$ Neurosurg 1982;57:99-107.

${ }^{23}$ Levy DE, Pike CI, Van Uitert RL. Delayed dissociation of cerebral blood flow and metabolism following stroke in gerbils. Neurology (Minneap) 1978; 28:378-9.

${ }^{24}$ Jones TH, Morawetz RB, Crowell RM, et al. Thresholds of focal cerebral ischaemia in awake monkeys. $J$ Neurosurg 1981;54:773-82.

${ }^{25}$ Astrup J, Symon L, Branston NM, Lassen NA. Cortical evoked potential and extracellular $\mathrm{K}^{+}$and $\mathrm{H}^{+}$at critical levels of brain ischaemia. Stroke 1977;8:51-7. 